\title{
Bone health in long-term survivors of acute lymphoblastic leukemia in children and ado-lecents. An assessment by peripheral quantitative computed tomography
}

\author{
Ronald Barr ${ }^{1}$, Dean Inglis ${ }^{2}$, Uma Athale ${ }^{2}$, Maciej Jaworski ${ }^{3}$, Troy Farncombe ${ }^{4}$, and \\ Christopher Gordon ${ }^{5}$ \\ ${ }^{1}$ McMaster Children's Hospital \\ ${ }^{2}$ McMaster University \\ ${ }^{3}$ Children's Memorial Health Institute \\ ${ }^{4}$ hamilton Health Sciences \\ ${ }^{5}$ Hamilton Health Sciences
}

April 9, 2021

\begin{abstract}
Background - Loss of bone mineral is a common concomitant of the treatment of acute lymphoblastic leukemia (ALL) due mainly to chemotherapy, especially with corticosteroids. Osteopenia/osteoporosis may persist long into survivorship. Measurement of bone mineral density (BMD) by dual energy X-ray absorptiometry is limited to two-dimensionality and cannot distinguish trabecular from cortical bone. Methods - A sample of 74 subjects, more than 10 years from diagnosis, underwent peripheral quantitative computed tomography (pQCT) at metaphyseal (trabecular bone) and diaphyseal (cortical bone) sites in the radius and tibia. pQCT provides three-dimensional assessment of bone geometry, density and architecture. Results - Similarities of average values in multiple metrics with those in healthy subjects obscured deficits in both trabecular and cortical bone, as well as bone strength, revealed by $\mathrm{Z}$ scores using an ethnically comparable sample of healthy individuals. Connectivity, a measure of bone architecture and a surrogate measure of bone strength, was lower in females than males. Survivors of standard risk ALL had greater connectivity in and more compact trabecular bone than high risk survivors who had received more intensive osteotoxic chemotherapy. There were no statistically significant differences in any of the metrics at any of the sites between subjects who had or had not a history of fracture, cranial irradiation or use of a bisphosphonate. Conclusions - These long-term survivors of ALL have somehat compromised bone health, but data in comparable healthy populations are limited. Longitudinal studies in larger and more ethnically diverse cohorts will provide greater insight into bone health in this vulnerable population.
\end{abstract}

\section{Introduction}

Acquisition of peak bone mineral mass, essential to bone health in adults, occurs during late childhood, adolescence and early adult life. This process can be disturbed by numerous factors including diet, disease, treatment and lifestyle. ${ }^{1}$ Such perturbation is well described in children and adolescents with acute lymphoblastic leukemia (ALL) before, during and after therapy. ${ }^{2}$ Important contributors to the outcome, osteopenia, are corticosteroids and methotrexate ${ }^{2,3}$ which are part of all treatment regimens for this disease. The most common method for determining bone mineral density (BMD) is dual energy X-ray absorptiometry (DXA) focused on the lumbar spine (LS). A limitation of DXA is the provision of two-dimensional measurement, so providing areal (a) BMD. Peripheral quantitative computed tomography (pQCT) allows the determination of volumetric (v) BMD, separate assessment of cortical and trabecular bone (not possible with DXA), and portability. In the overall study of bone health and body composition of long term survivors of ALL in early life,4 pQCT was undertaken in addition to DXA to enable a comprehensive evaluation of bone geometry, 
density and strength.5 The purpose was to improve understanding of the mechanisms resulting in enhanced risk of fracture and an element of the frailty syndrome which are well recognized in these survivors. 6 This report describes the pQCT component of the study. Such insights relating to bone structure should also inform strategies for amelioration and even prevention of osteopenia and its attendant morbidity.

\section{Patients and Methods}

Details of the design of the multi-element study of bone health and body composition have been published.4 From a cohort of children and adolescents who were diagnosed with ALL more than a decade earlier at McMaster Children's Hospital (MCH) a study sample was constructed (Fig 1). Treatment had been administered on protocols of the Dana Farber Cancer Institute Childhood ALL Consortium7 of which MCH had been a member since 1985 .

The current report focuses on bone geometry, density and architecture as assessed by pQCT performed with a Stratec XCT 2000 instrument (Stratec Medezintechnik, Pforzheim, Germany) with the Stratec Analysis Software v6.0 (Orthometrix Inc, White Plains, NY, USA). Measurements were made by a single experienced operator at the $4 \%$ sites of the length of the radius and tibia in the non-dominant limbs as well as at the $20 \%$ site of the radius and the $38 \%$ and $66 \%$ sites of the tibia, all with $0.2 \mathrm{~mm}$ voxel size and $2.2 \mathrm{~mm}$ slice thickness. The $4 \%$ (metaphyseal) sites represent trabecular bone and the proximal (diaphyseal) sites cortical bone.5 In order to emphasize metrics of high clinical importance and relevance to bone biology and biomechanics, including bone strength, as well as to allow comparisons with published reference values, analyses focused on 4 metrics at the metaphyseal sites - Total bone mineral content (BMC), Total vBMD, Trabecular BMC and Trabecular vBMD - and 6 metrics at the diaphyseal sites - Total BMC, Total vBMD, Cortical BMC, Cortical vBMD, Cortical Thickness and Polar Strength-Strain Index (SSI); a total of 10 selected from the many metrics provided by the Stratec software.

In addition to the standard volumetric density and geometry endpoints derived from the Stratec analysis software, measures of apparent trabecular architecture captured at the $4 \%$ metaphyseal sites were derived from the pQCT images. Specifically, the $4 \%$ metaphyseal images were post-processed with custom software developed by one of the authors.8 This builds on the work of CLG and colleagues, beginning 25 years ago. ${ }^{9-12}$ As reported, ${ }^{8}$ the software uses edge detection and thresholding steps to segment the trabecular bone and to quantify its structure. Fig 2 illustrates some of the image processing steps. The connectivity of the trabecular network is based on strut analysis in which the network is considered to consist of onedimensional struts (Fig 2A). ${ }^{9}$ A junction between three or more struts is defined as a node. A strut which is connected at one end but is free at the other is called a free end. Struts representing trabeculae running perpendicular to the image appear as points and are deemed to be isolated points. The connectivity of the bone architecture can also be assessed by quantifying the appearance of holes in the network due to loss of trabecular elements (Fig 2B). As more elements are lost, the average hole size reflects the increased mean distance between trabecular struts. ${ }^{11}$ Overall, from the 78 metrics available from post-processing, 7 were selected with respect to apparent trabecular bone architecture- Trabecular Thickness (mm), Trabecular Number $(1 / \mathrm{mm})$, Average Hole Size $\left(\mathrm{mm}^{2}\right)$, Maximum Hole Size $\left(\mathrm{mm}^{2}\right)$, Number of Free Ends per Unit Area (Free End Density $-\mathrm{mm}^{-2}$ ), Number of Isolated Points per Unit Area (Isolated Points Density $-\mathrm{mm}^{-2}$ ) and Connectivity Index (Number of Nodes per Unit Connected Network). A bone with high connectivity, and so strength (resistance to bending, torsion and compression), has a large number of nodes with few free ends and isolated points as well as small marrow pores (hole sizes). The custom program allows greater sensitivity to detect changes associated with perturbed bone metabolism that we have described in children with ALL. ${ }^{13}$

Of note, pQCT images can be acquired in a short period of time (10 minutes) with low radiation exposure (approximately $5 \mu \mathrm{Sv}$ ), ${ }^{14}$ equivalent to about 2 days of natural radiation. Comparisons were made with published data on healthy subjects data from relevant age groups using Stratec XCT 2000 and 3000 instruments. ${ }^{5,15-23}$ Comparative values for the $4 \%$ radius site are available from 4 publications, ${ }^{15}, 18,19,22$ and there are 4 reports on the $4 \%$ tibia site. ${ }^{16,19,21,23}$ Fewer were found for the diaphyseal sites - $20 \%$ radius, ${ }^{19}$ $38 \%$ tibia, ${ }^{17,20,21}$ and $66 \%$ tibia. ${ }^{16,23}$ Information from these sources were provided in Supplementary Tables 
1 and 2. There is no "gold standard" set of reference values for pQCT in children and adolescents. ${ }^{24}$ However, reference values for comparison at the $4 \%$ radius $^{22}$ and the $4 \%$ and $38 \%$ tibia $^{25}$ are available from a sample of more than 220 healthy Caucasian subjects, allowing the calculation of $\mathrm{Z}$ scores at these sites. The normative values for those aged 20-40 years are taken as equivalent to those in healthy 19 year old males and females. ${ }^{26}$ These data were obtained using Stratec equipment and software very similar to those used in the current study.

In light of the differences in body composition in this cohort according to sex ${ }^{27}$ the pQCT metrics were also examined separately in males and females. Data from pQCT are presented as means and standard deviations or as medians and ranges when appropriate. Subgroup analyses were undertaken on the 14 subjects who had received a bisphosphonate during therapy to correct osteoporosis, defined as an LS BMD Z score < $-2.0{ }^{28,29}$ Parametric data were compared with 2 tailed $t$ tests and non-parametric data by chi-squared tests. Correlations were measured with Pearson's correlation coefficient for parametric data and with Spearman's rho for non-parametric data. Comparisons of groups who did and did not receive a bisphosphonate were performed by Fisher's exact test. All analyses were performed with SPSS, version 24.

This study was approved by the Hamilton Integrated Research Ethics Board (project 10-508-5) which represents Hamilton Health Sciences, McMaster University and St. Joseph's Healthcare Hamilton. Informed consent was obtained from each participant.

\section{Results}

Study sample - a total of 74 survivors, almost all of them white Caucasian, formed the study sample. These subjects (40 males and 44 with standard risk [SR] ALL) were 13.5 - 38.3, median 21.1 years of age at the time of participation and $10.2-26.3$, median 15.1 years from diagnosis. High risk (HR) disease was defined by any one of the following - age $>10$ years, WBC $>50 \times 19^{9} / \mathrm{L}, \mathrm{T}$ cell phenotype, CNS involvement, mediastinal mass and $t(9 ; 22)$ translocation. The majority of the subjects $(n=51,69 \%)$ had received cranial irradiation, reflecting the era in which they were treated. All of the subjects underwent DXA and all but two had pQCT examination.

Total and trabecular BMC and trabecular vBMD were much lower at the (non-weight bearing) radial metaphysis than at the tibial metaphysis (Table 1), as in healthy subjects (Supplementary Tables 1 and 2) with which these values are closely similar. Cortical BMC and vBMD at the diaphyseal sites in both bones (Table 1) are also similar to published values (Supplementary Tables 1 and 2). Data from healthy subjects for cortical thickness are limited, with only one publication each at the $20 \%$ radius ${ }^{19}$ and $38 \%$ tibia, ${ }^{21}$ and two at the $66 \%$ tibia (Supplementary Tables 1 and 2). ${ }^{16,23}$ The mean values for this metric in the study sample were approximately the same as these published data; $2.33 \mathrm{v} 2.7,4.95 \mathrm{v} 5.0$ and $3.79 \mathrm{v} 4.1 \mathrm{~mm}$ at the $20 \%$ radius, $38 \%$ tibia and $66 \%$ tibia respectively. Published data for polar SSI are even more limited, with none at the $38 \%$ tibia. Polar SSI values at the $20 \%$ radius and $66 \%$ tibia in the study sample (Table 1 ) are quite similar to the published values (Supplementary Tables 1 and 2). ${ }^{15,18,22}$ Polar SSI, a measure of bone strength (resistance to bending and twisting), and combining geometry and density, was progressively higher in the diaphyseal shafts with increasing distance from the distal metaphyses at the wrist and ankle (Table 1). The $\mathrm{Z}$ scores provided by comparison with the reference population are given in Table 2 and reveal a different picture of the health of both metaphyseal and diaphyseal bone.

The metrics from post-processed images are displayed in Supplementary Table 3. Striking and highly significant differences were evident between males and females at the metaphyseal sites (Table 3). These differences were seen in the non-weight bearing radius but not in the weight bearing tibia, although the directionality was the same; males had greater BMC and vBMD with improved architecture - smaller average and maximum hole sizes with fewer free ends and isolated points, and so higher connectivity. Likewise, apparent trabecular thickness and number were higher in males.

A different profile was seen at all of the diaphyseal sites (Table 3). Males had significantly greater total and cortical BMC but females had higher total vBMD, though this was not statistically significant, and there were significantly higher values in cortical vBMD for females, probably reflecting their lower cortical 
thickness. Most importantly, the polar SSI (a measure of bone strength) was significantly greater in males than in females at all diaphyseal sites.

There were no significant differences in any of the metrics at metaphyseal sites related to age at examination. The only metric which revealed a statistically significant difference at the diaphyseal sites was cortical vBMD - at all three sites. The subjects were divided into those 13-20 (N=30) and those $>20$ years of age $(\mathrm{N}=42)$ and the younger group had lower values (20\% radius 1141.3 [55.7] vs 1218.3 [39.8], $\mathrm{p}<0.0001 ; 38 \%$ tibia 1149.7 [48.4] vs 1203.5 [22.2]. $\mathrm{p}<0.0001 ; 66 \%$ tibia 1089.5 [44.5] vs 1129.2 [26.6], $\mathrm{p}<0.0001$. This may relate to a smaller proportion of the younger subjects having attained peak bone mass.

With respect to ALL risk category, which is confounded by age at diagnosis - children 10 years of age and older are automatically high risk - there were no significant differences in BMC or vBMD, total or trabecular, at the metaphyseal sites between subjects who were SR and those HR at diagnosis, with the exception of Trabecular vBMD at the $4 \%$ radius, but there were notable differences in apparent bone architecture (Table 4). The SR group had smaller average and maximum hole sizes with fewer free ends and isolated points and so higher connectivity. Likewise, at both the $4 \%$ radius and $4 \%$ tibia the trabecular number was higher in SR than in HR subjects, reflecting more compact bone. At the diaphyseal sites there were statistically significant differences only with cortical vBMD; the HR subjects $(\mathrm{N}=29)$ having slightly higher values than those who had SR disease (20\% radius 1204.7 [52.8] vs 1173.8 [62.5], $\mathrm{p}=0.032 ; 38 \%$ tibia 1193.6 [43.9] vs 1172.7 [48.1], $\mathrm{p}=0.048 ; 66 \%$ tibia 1125.4 [28.0] vs 1104.4 [44.7], $\mathrm{p}=0.028)$, with no differences in cortical thickness.

There were no statistically significant differences in any of the metrics at any of the sites between subjects with $(\mathrm{N}=27)$ and those without $(\mathrm{N}=45)$ a history of fracture (two missing data), nor between those who had received a bisphosphonate $(\mathrm{N}=14)$ and those who had not $(\mathrm{N}=58)$; two missing data. No differences were observed between those who had $(\mathrm{N}=49)$ and those who had not $(\mathrm{N}=23)$ received cranial irradiation (two missing data) with respect to the metaphyseal bone metrics. At the diaphyseal sites there were no consistent differences between irradiated and non-irradiated subjects.

\section{Discussion}

In a detailed and comprehensive account of the Pediatric Official Positions of the International Society of Clinical Densitometry ${ }^{24}$ on quantitative computer tomography, Adams et al addressed the preferred choice of the non-dominant limb and the selection of sampling sites, including the $4 \%$ and $20 \%$ radius in addition to the $4 \%, 38 \%$ and $66 \%$ tibia as used in this study.

While the findings reported here suggest that, overall, the subjects, long term survivors of ALL in childhood and adolescence, exhibit mainly normal bone health, comparison with data from healthy populations has to be made with due consideration for important differences in their characteristics. For example, as reported by Leonard and colleagues at the Children's Hospital of Philadelphia $(\mathrm{CHOP})^{16}$ in a large group of healthy children, adolescents and young adults in the US, the racial composition of the study sample is critical. African Americans have higher values than Whites for numerous cortical measures at the $38 \%$ tibia which are reflected in a $13 \%$ greater average for the polar section modulus metric of bone strength. Similar differences exist between males and females. ${ }^{16}$ Comparable findings have been published from South Africa with bone strength measured by polar SSI. ${ }^{30}$ These authors have reviewed the international experience of the influence of ethnicity on bone. ${ }^{31}$ Consequently, the comparison of our study sample with a reference group of healthy subjects of the same ethnicity provides a more accurate assessment of their bone health. This reveals deficits in the mineralization of trabecular bone at the distal radial metaphysis in more than one third of subjects with a less consistent pattern in the corresponding tibia. Deficits in the mid-shaft of the tibia, except for cortical vBMD, are associated with reduced bone strength in almost one third of subjects.

Our findings of great total and trabecular vBMD in males than in females at the metaphyseal sites have been reported in healthy older adolescents. ${ }^{21,22}$

A seemingly counter-intuitive finding in the current study is the greater cortical thickness at the $38 \%$ than at 
the $66 \%$ tibial sites. However, in a remarkably detailed investigation conducted in 60 healthy young adults, pQCT was performed at 14 levels ranging in $5 \%$ increments from the $10 \%$ to the $80 \%$ sites on the tibia (and the fibula). ${ }^{32}$ The cortical thickness was substantially greater at the $40 \%$ than at the $65 \%$ tibial sites. Again, in concert with the current findings, the cross-sectional moments of inertia, measures of the stiffness of bone in relation to bending, were progressively greater proximally along the tibial shaft beyond the $30 \%$ site.

Although there are few reports of the use of pQCT to assess bone health in survivors of ALL in childhood, two are especially informative. Investigators in Manchester, UK studied 53 school-aged children and adolescents who had completed treatment, without cranial irradiation, 1.5 to 8.3 years earlier. ${ }^{33}$ Measurements were made at the $4 \%$ and $50 \%$ sites of the non-dominant radius and comparisons were made with close to 200 healthy subjects in the same age group; a cohort which grew to 500 in later years ${ }^{34}$ affording the opportunity to develop Z scores (though not applied earlier to the ALL group). The subjects who had survived ALL had lower trabecular but not total vBMD than the control group and the deficit was less the longer the interval from the completion of therapy in this cross-sectional study. Findings at the diaphyseal site suggested endosteal bone loss during treatment.

In rather the reverse order, the investigators at CHOP built a reference cohort of more than 650 individuals, affording the opportunity to provide $\mathrm{Z}$ scores for clinical populations. ${ }^{18}$ Subsequently they were able to study 50 survivors of ALL, ages 5-22 years, within two years of completing treatment which did not include cranial irradiation. ${ }^{35}$ Measurements were made at the $3 \%, 38 \%$ and $66 \%$ sites of the non-dominant tibia. Trabecular vBMD (at the $3 \%$ site) and cortical vBMD (at the $38 \%$ site) were significantly lower than in the reference group; no results were reported for total vBMD at either site. When studied one year later both measures showed improvement but were still significantly lower than in the reference group. The finding at the metaphysis is comparable to that reported by Brennan et al, ${ }^{13}$ but the diaphyseal measurements are not comparable because the sites are much different.

Our findings with post-processing of images point to greater connectivity in and more compact trabecular bone in SR than HR survivors. This may reflect the higher intensity of osteotoxic chemotherapy in the HR group.

The limitations of this cross-sectional observational study include a small sample size, although larger than the other published studies of pQCT in children who had ALL. Again, the subjects had a wide age span (15 years) and a wide range of time from diagnosis, averaging 15 years, although all were more than 10 years from that date. A longitudinal study would be useful, building on the experience of Leonard and colleagues. ${ }^{17}$ Addressing the lack of ethnic diversity in our subjects would require a much bigger sample size.

A particular strength of the study is the use of post-processing with custom software which expands information on apparent bone architecture compared to conventional pQCT. A prospective serial study of children with ALL, along their cancer journey from diagnosis to long term survivorship, using this technology would provide a detailed picture of osteotoxicity and recovery while offering opportunities for early therapeutic intervention to maximize ultimate bone health in this young population.

Conflict of Interest Statement. The authors have no conflict of interest to declare

\section{References}

1. Ward LM, Konji VN,Ma J. The management of osteoporosis in children. Osteoporosis Int 2016;7:21472149

2. Sala A, Barr RD. Osteopenia and cancer in children and adolescents. The fragility of success. Cancer 2007;109:1420-1431

3. Mostoufi-Moab S, Halton J. Bone morbidity in childhood leukemia. Epidemiology, mechanisms, diagnosis and treatment. Curr Osteoporosis Rep 2014;12:300-312

4. Barr R, Nayiager T, Gordon C, Marriot C, Athale U. Body composition and bone health in long-term survivors of acute lymphoblastic leukemia in childhood and adolescence: the protocol for a crosssectional cohort study. BMJ Open 2015;5: e006191 
5. Fonseca A, Gordon CL, Barr RD. Peripheral quantitative computed-tomography (pQCT) to assess bone health in children, adolescents, and young adults: A review of normative data. J Pediatr Hematol Oncol 2013;35:581-589

6. Ness KK, Krull KR, Jones KE et al. Physiologic frailty as a sign of accelerated aging among adult survivors of childhood cancer. J Clin Oncol 2013;31:4496-4503

7. Silverman LB, Stevenson KE, O'Brien JE et al. Long term results of Dana Farber Cancer Institute Childhood ALL Consortium protocols for children with newly diagnosed acute lymphoblastic leukemia (1985-2000). Leukemia 2010;24:320-324

8. Calder KM, Inglis D, MacIntyre NJ. Comparison of pQCT-based measures of radial bone geometry and apparent trabecular bone structure using manufacturer and in-house-developed algorithms. J Clin Densitom 2010;13:433-440

9. Gordon CL, Webber CE, Adachi JD, Christoforou N. In vivoassessment of trabecular bone structure at the distal radius from high-resolution computed tomography images. Phys Med Biol 1996; 41:495-508.

10. Gordon CL, Webber CE, Nicholson PS. Relation between image-based assessment of distal radius trabecular structure and compressive strength. Can Assoc Radiol J 1998;49:390-397.

11. MacIntyre NJ, Adachi JD, Webber CE. Gender differences in normal age-dependent patterns of radial bone structure and density. J Clin Densitom 1999;2:163-173.

12. MacIntyre NJ, Adachi JD, Webber CE. In vivo detection of structural differences between dominant and nondominant radii using peripheral quantitative computed tomography. J Clin Densitom 1999;2:413422 .

13. Atkinson SA, Halton JM, Bradley C, Wu B, Barr RD. Bone and mineral abnormalities in childhood acute lymphoblastic leukemia: influence of disease drugs and nutrition. Int J Cancer 1998; 11 Suppl: 35-39.

14. Gordon CL, Webber CE, Cottreau M. Radiation dose associated with peripheral quantitated computer tomography scanning in children. J Bone Miner Res 2003;18: S 302

15. Neu CM, Rauch F, Manz F et al. Modeling of cross-sectional bone size, mass and geometry at the proximal radius: a study of normal bone development using peripheral quantitative computed tomography. Osteoporos Int 2001;12:538-547.

16. Moyer-Mileur LJ, Quick JL, Murray MA. Peripheral quantitative computed tomography of the tibia: pediatric reference values. Clin Densitom 2008;11:283-294.

17. Leonard MB, Elmi A, Mostoufi-Moab S et al. Effect of sex, race and puberty on cortical bone and the functional muscle-bone unit in children, adolescents and young adults. J Clin Endocrinol Metab 2010;95:1681-1689.

18. Dowthwaite JN, Scerpella TA. Distal radius geometry and skeletal strength indices after peripubertal artistic gymnastics. Osteoporos Int 2011;22:207-216.

19. Pollock NK, Laing EM, Taylor RG et al. Comparisons of trabecular and cortical bone in late adolescent black and white females. J Bone Miner Metab 2011;29:44-53.

20. Wetzsteon RJ, Zemel BS, Shultz I et al. Mechanical loads and cortical bone geometry in healthy children and young adults. Bone 2011;48:1103-110.

21. Roggen I, Roelants M, Sioen I et al. Pediatric reference values for tibial trabecular bone mineral density and bone geometry parameters using peripheral quantitative computed tomography. Calcif Tissue Int 2015;96:527-533.

22. Jaworski M, Graff K. Peripheral quantitative computed tomography of the distal and proximal forearm in children and adolescents: bone densities, cross-sectional sizes and soft tissues reference data. J Musculoskelet Neuronal Interact 2018;18:237-247.

23. Jiang H, Yates CJ, Gorelik A, Kale A, Song Q, Wark JW. Peripheral quantitative computed tomography (pQCT) measures contribute to the understanding of bone fragility in older patients with low trauma fracture. J Clin Densitom 2018;21:140-147.

24. Adams JE, Engelke K, Zemel BS, Ward KA. Quantitative computer tomography in children and adolescents: The 2013 ISCD Pediatric Official Positions. J Clin Densitom 2014; 17: 258-274.

25. Jaworski M, Kobylińska M, Graff K. Peripheral quantitative computed tomography of the lower leg 
in children and adolescents: bone densities, cross-sectional sizes and muscular distribution reference data. J Musculoskelet Newuronal Interact 2020, in press.

26. Neu CM, Manz F, Rauch F, Merkel A, Schoenau E. Bone densities and bone size at the distal radius in healthy children and adolescents: a study using peripheral quantitative computed tomography. Bone 1; 28: 227-232.

27. Marriott CJC, Beaumont LF, Farncombe TH et al. Body composition in long-term survivors of acute lymphoblastic leukemia diagnosed in childhood and adolescence: A focus on sarcopenic obesity. Cancer 2018;124:1225-1231.

28. Saggese G, Baroncelli GI, Bertelloni S. Osteoporosis in children and adolescents: diagnosis, risk factors, and prevention. J Pediatr Endocrinol Metab 2001;14:833-859.

29. Di Iorgi N, Maruca K, Patti G, Mora S. Update on bone density measurements and their interpretation in children and adolescents. Best Pract Res Clin Endocrinol Metab 2018;32:477-498.

30. Micklesfield LK, Norris SA, Pettifor JM. Determinants of bone size and strength in 13-year-old South African children: the influence of ethnicity, sex and pubertal maturation. Bone 2011; 48: 777-785.

31. Micklesfield LK, Norris SA, Pettifor JM. Ethnicity and bone: A South African perspective. J Bone Miner Metab 2011; 29- 257-267.

32. Lüscher SH, Nocciolino LM, Pilot $\mathrm{N}$ et al. Differences in the cortical structure of the whole fibula and tibia between long-distance runners and untrained controls. Toward a wider conception of he biomechanical regulation of cortical bone structure. Front Endocrinol 2019; 10:833.

33. Brennan BMD, Mughal Z, Roberts SA et al. Bone mineral density in childhood survivors of acute lymphoblastic leukemia treated without cranial irradiation. J Clin Endocrinol Metab 2005; 90: 689694.

34. Ashby RL, Ward KA, Roberts SA, Edwards L, Mughal MZ, Adams JE. A reference database for the Stratec XCT-2000 peripheral quantitative computed tomography (pQCT) scanner in healthy children and young adults aged 6-19 years. Osteoporos Int 2009; 20: 1337-1346.

35. Moustofi-Moab S, Brodsky J, Isaacoff EJ et al. Longitudinal assessment of bone density and structure in childhood survivors of acute lymphoblastic leukemia without cranial radiation. J Clin Endocrinol Metab 2012; 97: 3584-3592.

Figure legends

Figure 1. Consort diagram

Figure 2. The distal pQCT scans are segmented with gradient and density threshold steps to reveal the trabecular pattern. For this distal tibia scan A, the trabecular pattern can be skeletonized to reveal its connectivity. The trabecular pattern can also be examined to reveal loss of individual trabecular elements which result in apparent holes in the bone network. As illustrated in B, a hole size analysis can be applied to the trabecular pattern

\section{Hosted file}

Table 1.pdf available at https://authorea.com/users/406883/articles/517371-bone-healthin-long-term-survivors-of-acute-lymphoblastic-leukemia-in-children-and-ado-lecents-anassessment-by-peripheral-quantitative-computed-tomography

\section{Hosted file}

Table 2.pdf available at https://authorea.com/users/406883/articles/517371-bone-healthin-long-term-survivors-of-acute-lymphoblastic-leukemia-in-children-and-ado-lecents-anassessment-by-peripheral-quantitative-computed-tomography

\section{Hosted file}

Table 3.pdf available at https://authorea.com/users/406883/articles/517371-bone-healthin-long-term-survivors-of-acute-lymphoblastic-leukemia-in-children-and-ado-lecents-anassessment-by-peripheral-quantitative-computed-tomography 


\section{Hosted file}

Table 4.pdf available at https://authorea.com/users/406883/articles/517371-bone-healthin-long-term-survivors-of-acute-lymphoblastic-leukemia-in-children-and-ado-lecents-anassessment-by-peripheral-quantitative-computed-tomography

FIGURE 1
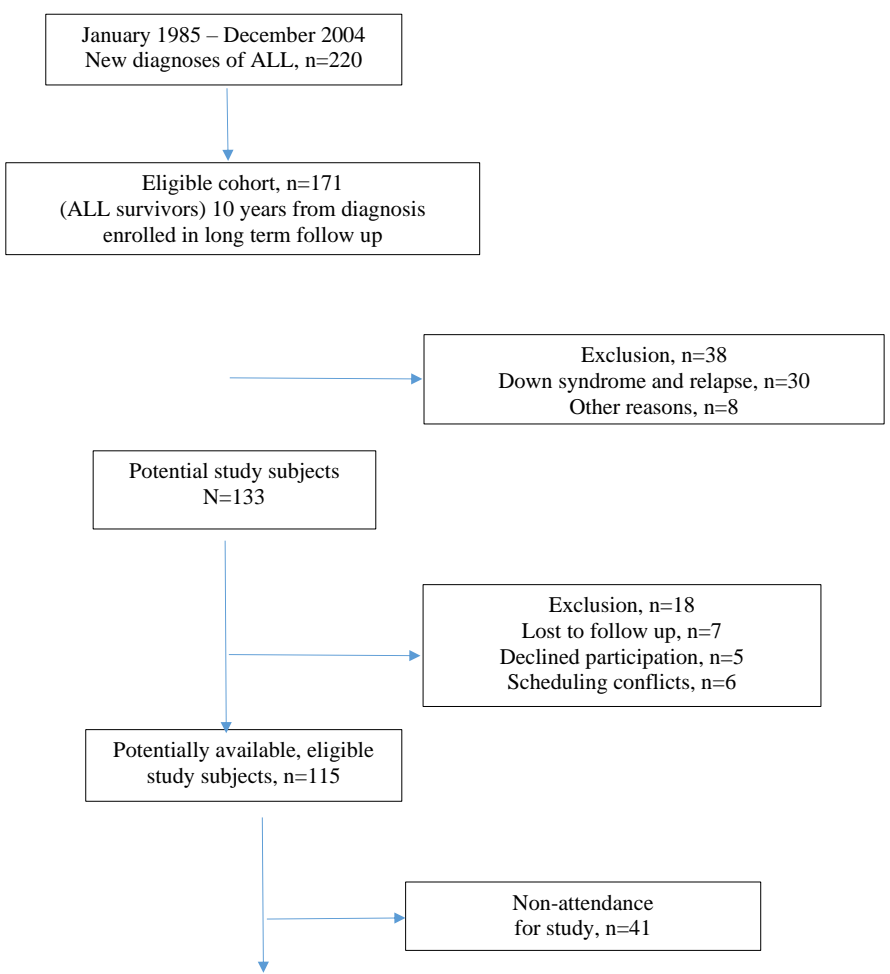

Final study cohort, n-74 
FIGURE 2.



B

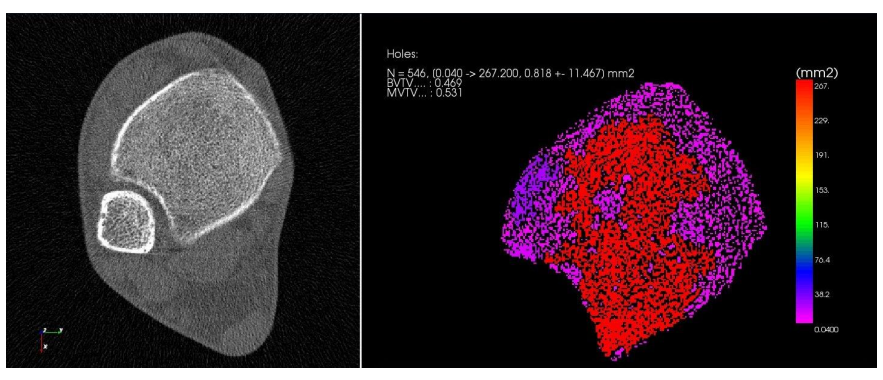

